\title{
PET-Saúde/Vigilância - UNISC: A relação com o ensino Odontológico
}

Tassia Silvana Borges*, Alexandre Daronco*, Charlene do Santos Silveira*, Eduardo Chaida Sonda*, Beatriz Baldo Marques**, Fabiana Battisti***, Cristiane Pimentel Hernandes Machado***, Alexandre Rauber****, Marcos Moura Baptista dos Santos*****, Lia Gonçalves Possuelo*******

* Bolsistas do PET Saúde/Vigilância - Universidade de Santa Cruz do Sul - UNISC

** Professora do Curso de Odontologia da Universidade de Santa Cruz do Sul - UNISC

*** Preceptoras do PET Saúde/Vigilância-Universidade de Santa Cruz do Sul- UNISC

**** Professor do Departamento de História e Geografia- Universidade de Santa Cruz do Sul - UNISC

***** Coordenador do Grupo PET-Vigilância em Saúde-Universidade de Santa Cruz do Sul - UNISC

****** Tutora Acadêmica do PET Saúde/Vigilância - Universidade de Santa Cruz do Sul - UNISC

\section{RESUMO}

O Programa de Educação pelo Trabalho para a Saúde - PET-Saúde/Vigilância - foi implantado na Universidade de Santa Cruz do Sul, no município de Santa Cruz do Sul, Rio Grande do Sul, com duas linhas de atenção: Atenção a Saúde do Trabalhador e Estratégias de Prevenção em Tuberculose. A magnitude da tuberculose explicita a necessidade de que não somente o médico, mas toda a equipe de saúde, incluindo o Cirurgião-Dentista, façam as identificações de possíveis casos de tuberculose e orientem corretamente seus pacientes. Material e Método: Por meio de reuniões semanais os acadêmicos foram divididos em grupos e realizaram o mapeamento de casos de Tuberculose (TB) nos anos 2000 a 2010. Concluída esta etapa foram realizadas capacitações para as equipes das Estratégias de Saúde de Família do município e palestras nas escolas da rede púbica de ensino além de campanhas na praça, no asilo municipal e no presídio regional, realizando busca de sintomáticos respiratórios. Resultados: Foram encontrados 523 casos de TB notificados no município. Nas capacitações foram mobilizadas 142 profissionais de saúde nas ESFs do município. Discussão: A distribuição, o modo de contágio e as formas de apresentação das doenças são de grande importância para o Cirurgião Dentista, sendo o PET Vigilância grande auxiliar na formação de tal profissional. Conclusão: Programas de ensino para saúde que orientem uma formação diferenciada vêm ao encontro das necessidades pungentes não somente da população como do Sistema de Saúde como um todo.

\section{DESCRITORES}

Odontologia. Vigilância. Tuberculose. Epidemiologia.

O Programa de Educação pelo Trabalho para a Saúde - PET-Saúde/Vigilância - foi implantado na Universidade de Santa Cruz do Sul-UNISC, no município de Santa Cruz do Sul-RS, com duas linhas de atenção: Atenção a Saúde do Trabalhador e Estratégias de Prevenção em Tuberculose. O PET-Saúde/ Vigilância tem como pressuposto a educação pelo trabalho, e é destinado a fomentar grupos de aprendizagem tutorial no âmbito da Vigilância em Saúde, caracterizando-se como instrumento para qualificação em serviço dos profissionais da saúde, bem como de iniciação ao trabalho e vivências direcionadas aos estudantes dos cursos de graduação desta área. Tal programa direciona suas atividades de acordo com as ações do Sistema Único de Saúde-SUS, tendo em pers- 
pectiva as necessidades dos serviços como fonte de produção de conhecimento e pesquisa nas instituições de ensino. Fazem parte do PET-Saúde/Vigilância-Tuberculose, acadêmicos dos cursos de Medicina, Enfermagem, Nutrição, Fisioterapia, Farmácia e Odontologia.

A tuberculose (TB) é uma doença de amplitude mundial, cujo principal agente etiológico é o Mycobacterium tuberculosis. ${ }^{5}$ A Organização Mundial da Saúde (OMS) estima que ocorreram em torno de 9,4 milhões de casos de TB em todo o mundo no ano de 2009. ${ }^{7}$ A mortalidade associada a esta doença ainda registra valores muito elevados: 1,7 milhões de mortes no ano de 2009, o que representa um total de 4.700 mortes/dia. O Brasil é um dos 22 países responsáveis por $80 \%$ dos casos de TB do mundo. ${ }^{3}$

O Estado do Rio Grande do Sul (RS) em 2009 atingiu uma taxa de incidência de 48 casos / 100.000 habitantes. A cidade de Porto Alegre é uma das capitais brasileiras que apresenta as maiores taxas de incidência, chegando a 118 casos/100.000 habitantes. Santa Cruz do Sul é um município da região central do estado do RS e está entre os 15 municípios prioritários para o controle da TB, onde a incidência chega a 50 casos/100.000 habitantes, além de apresentar taxas de cura menores que as recomendadas pelo Ministério da Saúde. ${ }^{4}$

A magnitude da patologia explicita a necessidade de que não somente o médico, mas toda a equipe de saúde, incluindo o Cirurgião-Dentista, façam a identificação de possíveis casos de tuberculose e orientem corretamente seus pacientes. Não obstante, o desconhecimento sobre a tuberculose leva o CirurgiãoDentista a não reconhecer ou não orientar adequadamente aqueles que atende. Tal fato advém de sua formação acadêmica, na qual tal patologia não é abordada. Outro ponto que merece destaque é a relevância que a epidemiologia tem para profissionais de saúde, podendo auxiliar na atuação em prevenção de doenças pelo conhecimento de suas características. ${ }^{2}$

O presente artigo tem por objetivo, através de relato acerca das atividades realizadas por acadêmicos do PET-Saúde/Vigilância, demonstrar como tal projeto pode ampliar a formação em Odontologia tanto no que tange um maior conhecimento a respeito de uma doença infecciosa de suma relevância quanto no entendimento da epidemiologia como ferramenta de prevenção e promoção de saúde.

\section{MATERIAIS E MÉTODOS}

Realizam-se reuniões semanais para discutir o an- damento do trabalho e propor novas atividades dentro do grupo PET-Saúde/Vigilância, sendo que em algumas destas é realizada divisão do grande grupo em equipes menores para executar determinadas tarefas. A primeira tarefa proposta foi o mapeamento de todos os casos de TB desde o ano 2000 até o ano de 2010 no município de Santa Cruz do Sul, buscando os dados no local de referência para o tratamento da TB e no Sistema de Informações sobre Agravo de Notificação do Ministério da Saúde (SINAM). Após, realizou-se o geoprocessamento dos casos utilizando o sistema software Google Earth. Na etapa seguinte os padrões de pontos de ocorrência dos eventos foram inseridos no AutoCadMap sobre o mapa base de logradouros e bairros fornecido pela Prefeitura Municipal e posteriormente os pontos marcados foram exportados para o TerraView ver. 3.5.0, um Sistema de Informações Cartográficas (SIG), desenvolvido pelo Instituto Nacional de Pesquisas Espaciais (INPE). No TerraView foram realizadas as análises geoespaciais e a produção dos mapas temáticos. Este projeto foi previamente submetido à apreciação e aprovação do Comitê de Ética em Pesquisa da Universidade de Santa Cruz do Sul e aprovado sob protocolo número 2764/10.

Finalizada tal atividade, foram localizados os bairros do município com maior incidência de TB. $\mathrm{Na}$ etapa seguinte, realizaram-se capacitações para equipes das ESFs do município, abrangendo também o município de Venâncio Aires com capacitação para os Agentes de Saúde do Programa de Agentes Comunitários de Saúde (PACS). As capacitações se realizaram em dias alternados, sendo que todo o material foi confeccionado pelos acadêmicos, como slides para as apresentações, folders e cartilhas explicativas para os ACS. Cada capacitação foi previamente agendada com as enfermeiras responsáveis pela equipe em horários adequados para as mesmas e para os acadêmicos.

Outra importante atividade desenvolvida pelo grupo foram as palestras nas escolas públicas de ensino fundamental e médio do município com o intuito de melhorar a qualidade de informação referente à TB entre crianças e adolescentes. Estas palestras também foram preparadas pelos próprios bolsistas e ministradas dependo da disponibilidade destes e das escolas. Para estas atividades foram disponibilizados os microscópios para visualização do Bacilo de Koch, correlacionando assuntos vistos em sala de aula com aspectos importantes sobre a tuberculose.

Foram realizadas campanhas no município no 
intuito de divulgar e conscientizar a população sobre a importância da TB e a busca pelos sintomáticos respiratórios. Para estas atividades foram selecionados locais estratégicos, como a praça principal da cidade, o asilo municipal e o presídio regional. Nestas ocasiões foram distribuídos os folders explicativos da TB e feita a busca de sintomáticos respiratórios identificando indivíduos com tosse persistente por mais de 3 semanas. A coleta do escarro para realização da baciloscopia foi também realizada.

\section{RESULTADOS}

Foram encontrados nestes 10 anos 523 casos notificados de TB no município, compreendendo área rural e urbana. A primeira parte do estudo conseguiu levantar dados importantes como identificação do local de maior prevalência como sendo o Presídio Regional da cidade de Santa Cruz do Sul-RS.

Munidos de informações acerca da epidemiologia da Tuberculose na cidade, os acadêmicos realizaram capacitações com as equipes de saúde. Desde o mês de março de 2011 tais capacitações são realizadas com profissionais Médicos, Odontólogos, Auxiliares de Saúde Bucal, Enfermeiras, Técnicas de Enfermagem e Agentes Comunitárias de Saúde. O PET-Saúde/Vigilância teve como resultado das capacitações a mobilização de 142 pessoas das dez ESFs do município e 88 alunos nas capacitações realizadas nas escolas.

A busca de sintomáticos respiratórios em campanhas na praça principal da cidade, no asilo municipal e no presídio regional foram feitas realizando-se 30 baciloscopias entre os sintomáticos respiratórios, todas negativas. O resultado alcançado pelo PET-Saúde/Vigilância-Tuberculose está sendo divulgado nos jornais de circulação local, em programas de televisão, em congressos com resumos elaborados pelos acadêmicos e em produções de artigos com os resultados obtidos nas pesquisas.

Com as capacitações percebemos que os profissionais começaram a modificar a forma de ver a tuberculose nos dias atuais. Na prática do Cirurgião- Dentista pouco esta doença é comentada ou lembrada, o que fez os dentistas do município lançarem a proposta de capacitações específicas para os mesmos, trazendo como sugestão modificar o prontuário dos pacientes, o qual não consta perguntas sobre os sintomas da TB. Lembramos que a TB, AIDS, Hepatite e Herpes são doenças infecto-contagiosas associadas a profissão do Cirurgião-Dentista. Por este motivo, devemos saber que as medidas de controle de infecção devem ser praticadas com rigor. $\mathrm{O}$ risco de contrair doenças e infecções em atendimentos odontológicos está diretamente ligado a não observância de preocupações universais de biossegurança, temos algumas medidas a serem tomadas:

- Em relação ao paciente: Anamnese.

- Em relação à equipe: Uso de Equipamento de Proteção Individual.

Estas observações foram levantadas para reafirmar a importância do Cirurgião Dentista frente a situações como estas.

\section{DISCUSSÃO}

O entendimento epidemiológico desenvolvido a partir da pesquisa permite ao acadêmico compreender a importância de ações de prevenção, ${ }^{5}$ não somente sobre a doença na qual o trabalho foi pautado, mas também no que se refere a qualquer patologia. A distribuição, o modo de contágio e as formas de apresentação das doenças são de grande importância para o Cirurgião-Dentista, sendo o PET-Saúde/Vigilância grande auxiliar na formação de tal profissional.

As capacitações que se realizam para equipes de saúde trazem o ambiente acadêmico para próximo da realidade de trabalho que o futuro profissional irá vivenciar. Tal aproximação permite a formação de uma consciência crítica ampla, capaz de compreender não somente o funcionamento do atendimento no serviço de saúde, como também peculiaridades do mesmo. Dentre os aspectos que interferem no desenvolvimento das ações de controle das doenças pelas equipes de profissionais que compõem a rede básica de saúde, apontam-se a qualificação profissional. Mesmo com a implantação da ESF, tem se observado uma inabilidade dos profissionais para abordar a problemática de algumas questões nos municípios brasileiros. ${ }^{1}$ Com programas de educação semelhantes ao PET-Saúde, tal vicissitude pode ser superada em vista do trabalho desenvolvido com as equipes de saúde e à formação de profissionais com formação mais direcionada às necessidades da realidade.

\section{CONClusões}

A Odontologia está intimamente relacionada a este tema, pois a tuberculose é uma doença presente em nosso meio, transmitida pelo ar, que tem sintomas característicos e que se tratada corretamente tem cura. A proposta que surgiu dos dentistas do município é uma prova de que cada vez mais devemos nos envolver com esta temática. O PET-Saúde/Vigilância é uma forma de integrar toda a comunidade acadê- 
mica e a rede de serviço, fazendo com que todos participem incessantemente na busca pela proteção, promoção e recuperação da saúde.

O ensino odontológico necessita de programas de educação para formação de profissionais que atuem além do simples agir em lesões bucais, é preciso uma visão ampliada do ser humano. Isto inclui a compreensão de algumas doenças sistêmicas como a tuberculose e habilidade para lidar com ferramentas e dados epidemiológicos.

Por conseguinte, o PET-Saúde/Vigilância e programas de ensino para saúde que orientem uma formação diferenciada vêm ao encontro das necessidades pungentes não somente da população como do Sistema de Saúde como um todo. Da mesma maneira, é importante frisar que programas como PET-Saúde impelem o estudante a não somente resgatar seu conhecimento, como a buscar novos saberes. Embora manifestações bucais da tuberculose decorram usualmente da implantação do bacilo existente no escarro resultante da expulsão de bactérias pela tosse, sendo a linha mediana do dorso da língua, o palato e lábios como sítios mais prevalentes, ${ }^{5}$ tal conhecimento, embora assaz relevante, é desconhecido para grande parcela de profissionais odontólogos. Graças a pesquisa realizada, acadêmicos da área puderam não somente lapidar seu saber, como desenvolver novos conhecimentos a partir das indagações que o trabalho suscitou.

\section{ABSTRACT}

\section{PET-Health/Surveillance - UNISC: Relationship with dental teaching}

The Education through Health Work Program / Surveillance (PET-SAÚDE/Vigilância) was implemented at the University of Santa Cruz do Sul in the municipal district of Santa Cruz do Sul, Rio Grande do Sul, guided by two lines of attention: Attention to Worker's Health and Strategies of Tuberculosis Prevention. The magnitude of tuberculosis clearly expresses the need for not only the doctor, but also the whole health team, including the Dentist, to identify possible cases of tuberculosis and guide patients correctly. Method: Students got together in weekly meetings and were divided into groups; they performed the mapping of tuberculosis cases (TB) in the years 2000 to 2010 . Once this stage was concluded, training sessions were held for the Family Health Strategy (ESF) teams of the municipal district, and lectures were given in the schools of the public education network, in addition to campaigns in the square, at the municipal asylum and in the regional prison, in search of people exhibiting symptomatic breathing. A total of 523 cases of TB were found in the municipal district, and were notified. In the training sessions, 142 ESF health professionals were engaged in the municipal district. Discussion: The distribution, the infection and the manner of presentation of diseases are of great importance for Dentists, and PET/ Surveillance is of great help in the training of a professional. Conclusion: Health-oriented teaching programs that guide a distinctive form of training for health professionals address the acute needs not only of the population but also of the Health System as a whole.

\section{DESCRIPTORS}

Dentistry. Surveillance.Tuberculosis. Epidemiology. "

\section{REFERÊNCIAS BIBLIOGRÁFICAS}

1. BRASIL, MINISTÉRIO DA SAÚDE. Secretaria de Gestão do Trabalho e da Educação na Saúde. Curso de Formação de Facilitadores de Educação Permanente em Saúde. Brasília: Ministério da Saúde, 2005.

2. Fletcher, R. H.; Fletcher, S. W. Epidemiologia clínica: elementos essenciais. 4. ed. Porto Alegre: Artmed, 2006.

3. Manual de Recomendações para o Controle de Tuberculose no Brasil [Internet]. Brasil: Ministério da Saúde. 2010 - [cited 2011 Jan 13]. Avaible from: http://www.ncbi.nlm.nih.gov/ books/NBK7273/.

4. PECT. Programa Estadual de Controle da Tuberculose do Estado do Rio Grande do Sul. Secretaria Estadual da Saúde. 2010

5. Sociedade Brasileira de Pneumologia e tisiologia. III Diretrizes para Tuberculose da Sociedade Brasileira de Pneumologia e Tisiologia. J Bras Pneumol. 2009; 35(10):1018-1048.

6. Volkweis, R.M. et al. Lesões bucais manifestadas em pacientes aidéticos e tuberculosos, relacionadas com a contagem celular cd4+ / cd8+. PGR-Pós-Grad Rev Fac Odontol São José dos Campos, v.4, n.3, set./dez. p. 74-82. 2001.

7. WHO. Global Tuberculosis Control [Internet]. Geneva: World Health Organization. 2010 - [cited 2011 Jan 10]. Avaible from: http://whqlibdoc.who.int/publications/2010/9789241564069_eng.pdf 